\title{
Téoros
}

Revue de recherche en tourisme

\section{Le tourisme et les lesbiennes}

Recherche de soi, recherche d'un ailleurs

\section{Line Chamberland}

Volume 19, numéro 2, été 2000

Le tourisme des gais et des lesbiennes

URI : https://id.erudit.org/iderudit/1071959ar

DOI : https://doi.org/10.7202/1071959ar

Aller au sommaire du numéro

Éditeur(s)

Université du Québec à Montréal

ISSN

0712-8657 (imprimé)

1923-2705 (numérique)

Découvrir la revue

Citer cet article

Chamberland, L. (2000). Le tourisme et les lesbiennes : recherche de soi, recherche d'un ailleurs. Téoros, 19(2), 16-21. https://doi.org/10.7202/1071959ar d'utilisation que vous pouvez consulter en ligne.

https://apropos.erudit.org/fr/usagers/politique-dutilisation/ 


\section{$+\ldots$ \\ LE TOURISME \\ ET LES LESBIENNES \\ RECHERCHE DE SOI, RECHERCHE D'UN AILLEURS}

\section{Line Chamberland}

Existe-t-il des pratiques touristiques spécifiques aux lesbiennes? Voyagent-elles beaucoup? Privilégient-elles le tourisme urbain, sexuel, culturel ou les escapades dans la nature? Sont-elles attirées vers des destinations particulières ou des lieux qui revêtent à leurs yeux une signification symbolique? Une fois parvenues à bon port, entrent-elles en contact avec les sous-cultures gaies et lesbiennes locales? Nouent-elles des relations à l'étranger afin d'enrichir leur réseau social ? Comment leurs activités touristiques se démarquent-elles de celles des hommes gais? Sont-elles affectées par les variables sociologiques (classe sociale, origine culturelle, génération, niveau d'éducation) qui modèlent les autres facettes de l'expérience du lesbianisme (Chamberland, 1996 ; Faderman, 1991 ; Lewin, 1996 ; Newton, 1993) ?

L'état actuel des recherches au Québec ne permet pas de répondre de manière appropriée à toutes ces questions, mais il en confirme la pertinence. De récents ouvrages se sont intéressés à l'émergence des communautés gaies et lesbiennes à Montréal depuis la Seconde Guerre mondiale jusqu'aux années 1990 (Higgins, 1997, 1999 ; Demczuk et Remiggi, 1998 ; Hildebran, 1997). Tout en retraçant le développement des sous-cultures homosexuelles, ils ont documenté leur inscription dans l'espace urbain, notamment par l'utilisation de lieux publics à des fins de rencontres sociales et sexuelles, de même que la création, depuis les années 1970, d'espaces commerciaux et associatifs, tantôt mixtes, tantôt distincts pour les gais et les lesbiennes. Il en ressort que depuis plusieurs décennies déjà, la vie homosexuelle à Montréal jouit d'un dynamisme et d'une visibilité qui fait de la métropole un pôle d'attraction touristique et résidentielle pour ceux et celles qui proviennent des autres régions du Québec, sans compter les visiteurs étrangers ${ }^{1}$. Ces dernières années, les médias et les politiciens ont découvert le pouvoir économique des hommes homosexuels dont «l'argent rose » contribue à alimenter l'industrie touristique locale ${ }^{2}$. Cependant, le phénomène du tourisme gai demeure peu étudié et, pour ce qui est des lesbiennes, pratiquement inexploré ${ }^{3}$.

Conséquemment, dans cet article je poursuis un objectif très modeste, soit celui de formuler des réponses partielles aux questions soulevées précédemment, m'appuyant sur des témoignages datant des années 1950 aux années 1970. Ma réflexion s'articule autour d'une perspective théorique qui appréhende l'expérience lesbienne sous l'angle de la régulation sociale et sexuelle par les diverses institutions et en tant que forme de résistance à cette régulation. Les institutions judiciaire, religieuse et psychomédicale remplissent à cet égard des fonctions distinctes et complémentaires dont l'agencement se modifie selon le contexte socio-historique. Ainsi, pendant la majeure partie de la période considérée, l'appareil judiciaire intervient pour réprimer les conduites sexuelles déviantes et, plus généralement, les manifestations visibles du lesbianisme dans la sphère publique. Mais c'est principalement à travers le contrôle religieux que s'effectue la régulation sexuelle jusqu'au milieu des années 1960. En effet, avant la Révolution tranquille, l'Église catholique, outre ses interventions dans le champ du spirituel, chapeautait et légitimait un ensemble de fonctions régulatrices exercées par d'autres institutions, surtout auprès des femmes. Le contrôle religieux s'avère donc celui dont la portée, directe et indirecte, était la plus étendue : il rejoignait l'ensemble des femmes et cela, dès leur plus jeune âge puisque la morale catholique était relayée par la famille et l'école; il s'étendait à plusieurs sphères d'activités où celles-ci travaillaient en tant que salariées, religieuses ou bénévoles ; enfin, il les touchait dans leur vie privée et dans leur conscience individuelle. De son côté, l'appareil psychomédical n'occupait encore qu'une position subordonnée, quoique son influence commença à crôtre à partir des années 1960. En pratique, la régulation sexuelle s'effectue à deux niveaux interreliés, interpersonnel et institutionnel, qui s'imbriquent l'un dans l'autre pour mouler la sexualité des femmes et contrôler les comportements s'écartant des normes. Par exemple, face à une conduite déviante, 1 'entourage immédiat s'appuie sur les discours normatifs afin d'exercer des pressions et justifier des mesures punitives, ou encore, si cela ne suffit pas, il fait appel aux autorités institutionnelles en place, lesquelles pourront imposer des sanctions plus graves ${ }^{4}$. En voulant échapper à la contrainte à l'hétérosexualité qui pèse sur toutes les femmes, les lesbiennes sont confrontées non seulement aux interdits sexuels, mais également aux limites qui découlent de leur position subordonnée à l'intérieur des 
rapports sociaux de sexe. En d'autres termes, comparativement aux hommes, leurs déplacements sont généralement plus restreints et surveillés, leur pouvoir économique, moindre ; bref, elles jouissent d'une moins grande autonomie personnelle. Dans ce texte, j'avance des pistes à approfondir en matière de la mobilité spatiale et géographique des lesbiennes, dans un cadre touristique ou autre, à savoir que cette mobilité est relativement intense, qu'elle coïncide souvent avec la recherche d'une plus grande liberté et qu'elle nourrit la construction de leur identité lesbienne.

\section{LES ANNÉES 1950 ET 1960 : LE TOURISME COMME ÉVASION ET COMME RECHERCHE IDENTITAIRE}

Les propos qui suivent s'appuient sur des entrevues réalisées auprès de vingt-quatre femmes, très majoritairement francophones et ayant reçu une éducation catholique, dont les expériences sexuelles et amoureuses avec d'autres femmes ont été vécues dans le contexte montréalais de cette époque ${ }^{5}$. Ce n'est qu'au fil des entretiens que la question des voyages et, plus généralement, de la mobilité spatiale est apparue comme une dimension importante de leur vie. Les données recueillies à ce propos ne sont pas exhaustives, mais elles suffisent pour faire ressortir certaines pratiques communes à plusieurs d'entre elles ainsi que les motivations qui les soustendent. En général, il m'a semblé que la majorité des interviewées se déplaçaient beaucoup, surtout si l'on tient compte du peu d'autonomie spatiale consentie aux femmes à cette époque lorsqu'elles n'étaient pas accompagnées par un homme ${ }^{6}$. Tout d'abord, on note qu'au moins une dizaine d'entre elles possédaient une automobile, surtout parmi les enseignantes et les autres professionnelles qui en avaient les moyens. D'autres en empruntaient une à leur famille ou se déplaçaient en compagnie de leur amante ou encore avec des amis gais et lesbiennes. Plusieurs d'entre elles avaient appris jeunes à conduire. En facilitant leurs déplacements, l'automobile accroissait leur mobilité spatiale et leur permettait de se livrer à toutes sortes d'activités : cours du soir, sorties, voyages courts de fins de semaine et de plus longue durée pendant les vacances d'été, etc. De toute évidence, la multiplication des activités extérieures correspondait à une stratégie d'éloignement du domicile résidentiel, qu'il soit celui de leurs parents ou le leur. Elles échappaient ainsi à la surveillance de l'entourage immédiat (famille et voisinage), tout en justifiant leurs nombreuses absences. Au besoin, l'automobile permettait de se rendre chez l'amante quand celle-ci habitait à l'extérieur de Montréal. Incidemment, il arrivait que le véhicule serve d'espace sexuel comme l'illustre l'histoire rocambolesque de l'une d'elles qui vivait ses toutes premières expériences sexuelles avec une autre femme en faisant du « parking » sur le bord du fleuve, quand un policier les a surprises en très petite tenue... Sa partenaire du moment a heureusement réussi à l'embobiner pour qu'il les laisse tranquilles.

La très grande majorité des interviewées pratiquaient régulièrement des activités touristiques au Québec et à l'étranger, avec leur amante, leurs amies lesbiennes ou encore, quoique rarement, des amis gais. Parmi les plus courantes, on trouve le camping d'été, le séjour dans un chalet, loué ou appartenant à la famille d'origine, dans des auberges ou autres lieux vacanciers reconnus, tout dépendant des goûts, des possibilités et des revenus. Un autre aspect à considérer est le maintien d'une façade légitime aux yeux des proches, surtout pour celles qui habitaient encore avec leurs parents. Par exemple, une interviewée raconte qu'elle participait à une troupe de danse folklorique avec son amante et deux amis gais. Ils partaient régulièrement tous les quatre en tournées ou simplement par plaisir. En présentant l'image de deux couples qui voyageaient ensemble, le change était donné.

L'éloignement procure un certain anonymat : même s'il faut censurer ses paroles et ses gestes, le risque d'un étiquetage comme lesbienne apparaît réduit et les conséquences moins dramatiques puisqu'il n'est pas le fait de personnes en autorité ou qui ont un pouvoir sur leur vie, leur emploi, leur réputation personnelle. Outre la marge de liberté ainsi acquise, l'activité touristique permet l'accès à un espace privé plus ou moins grand, plus ou moins fermé à l'intrusion d'autrui, où la sexualité peut s'exprimer dans une relative intimité. Le chalet isolé constitue un idéal à cet égard, ce qui explique sans doute sa popularité. Selon certains témoignages, la distanciation psychologique favorisait également l'épanouissement du désir sexuel. Par

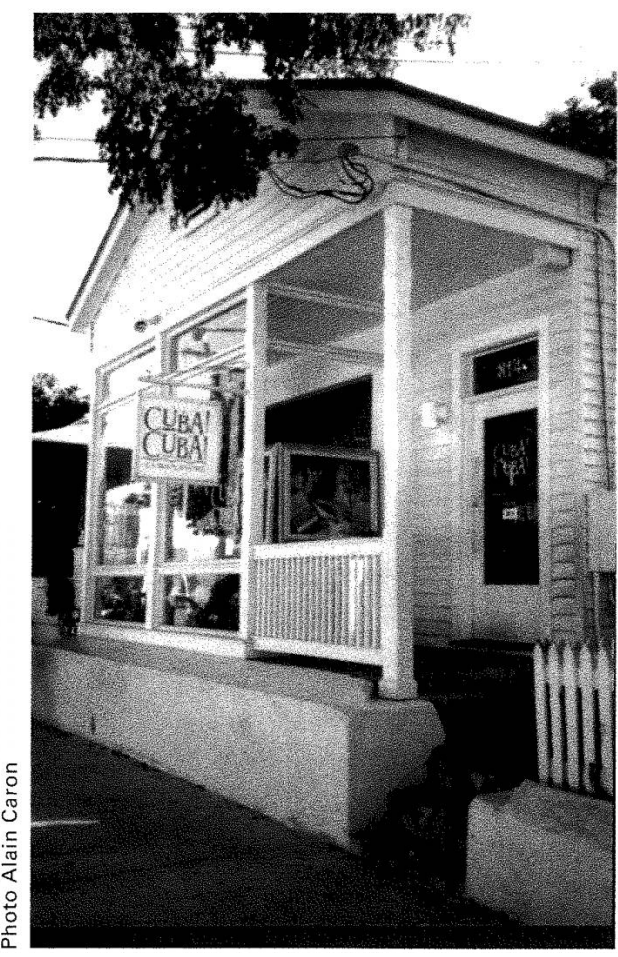

Key West, Floride. Les lesbiennes s'y rendent pour le WomenFest.

exemple, une femme qui n'habitait pourtant plus chez ses parents depuis quelques années se rappelle avoir « explosé sexuellement » dans un motel de la Gaspésie, bien loin de chez elle. Il faut rappeler ici l'extrême sévérité des interdits sexuels qui étaient intériorisés à des degrés divers.

Avoir accès à un espace privé et éloigné ne revêt pas la même importance d'une génération à l'autre. Ainsi, pour les interviewées nées avant la Seconde Guerre mondiale, quitter le domicile familial sans raison légitime (se marier ou entrer en religion) n'allait pas de soi. Il fallait alors recourir à divers stratagèmes pour sortir de l'orbite de la famille. Outre leur finalité propre, les activités touristiques contribuaient donc à accroître la marge d'autonomie personnelle tout en évitant de rompre avec la famille d'origine. Chez les plus jeunes, le besoin d'une « chambre à soi » était relativement facile à combler car elles quittaient très tôt le domicile familial pour aller vivre en appartement. Pour toutes, s'éloigner de Montréal facilitait l'aménagement de l'identité lesbienne en accroissant le degré d'anonymat et cela, même lorsqu'elles devaient tenir compte des contraintes du nouveau contexte dans lequel elles se trouvaient pour éviter de se faire repérer en tant que «femme-auxfemmes ». 
Des variations s'observent également selon le degré de visibilité sociale en tant que lesbiennes. Les plus cachées, qui censuraient constamment leurs gestes et leurs paroles afin de ne pas éveiller les soupçons de l'entourage, ne se sentaient à l'aise d'extérioriser leur identité sexuelle que dans des lieux clos et distants, ou encore dans une ville ou un pays étranger, où ne pouvaient les accompagner, outre leur amante, que des amies triées sur le volet. Elles profitaient alors de cette occasion unique pour échanger des confidences sur leur vie amoureuse, commenter films et livres sur l'homosexualité (qu'elles avaient vus ou lus dans le secret), discuter de leurs préférences sexuelles, bref pour concrétiser ce qu'elles vivaient habituellement dans le silence. Les voyages lointains contrastaient fortement avec l'obligation quotidienne de mener une double vie :

\section{C'était vraiment juste en vacances qu'on arrivait à vivre bien. Je me sentais heureuse rien qu'en voyage parce que c'était à l'extérieur et personne ne nous connaissait. Je me sentais parfaitement libre. La France était beaucoup plus ouverte aux gens, aux mours... à des choses différentes. On n'a jamais eu de remarques négatives en voyage, jamais. C'était un désespoir de re- venir en septembre et de recommen- cer à ne pas parler, à ne pas vivre... C'était pénible, pénible.}

La distanciation géographique se révèle une stratégie efficace pour échapper au contrôle social. En effet, dans une grande ville comme Montréal, les mécanismes institutionnels de régulation sexuelle ne s'avéraient efficaces que s'ils étaient relayés par l'entourage immédiat (famille, travail...), lequel surveillait et sanctionnait les conduites. Le tourisme constituait alors une façon d'échapper au regard des proches. Les seules qui ne recouraient pas à cette manœuvre sont celles qui, à la fois, étaient plus visibles en tant que lesbiennes, donc déjà étiquetées, et n'avaient que de maigres moyens financiers, étant confinées à des occupations peu ou pas qualifiées. Précisons-le : ces deux caractéristiques allaient souvent de pair.

Tout dépendant des destinations, l'activité touristique fournissait également l'occasion d'explorer des univers homosexuels et lesbiens, réels ou imaginaires, en toute

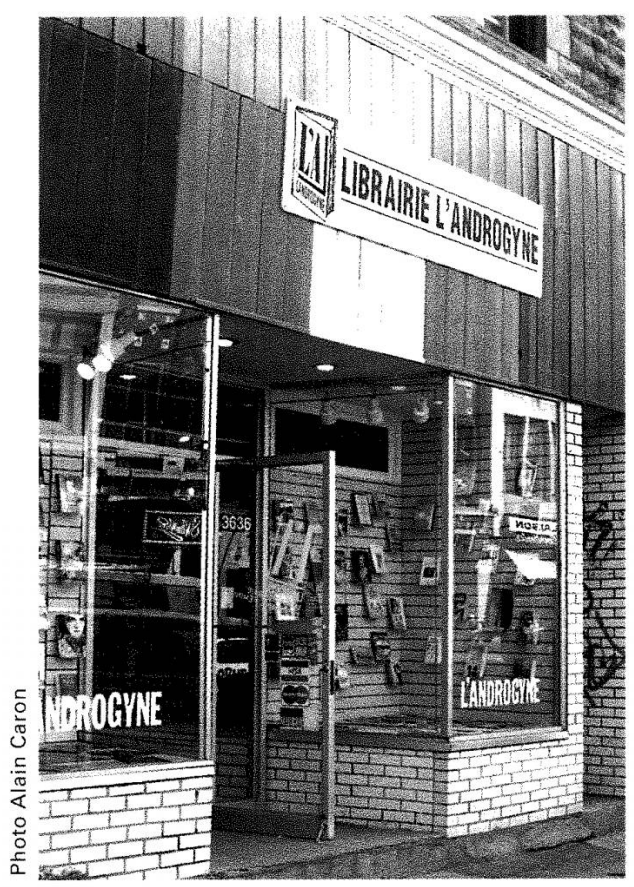

La librairie gaie et lesbienne montréalaise L'Androgyne, sur le boulevard Saint-Laurent.

sécurité. Avant les années 1970, le lesbianisme était pratiquement tabou : peu visible comme phénomène social de l'avis de toutes les lesbiennes interrogées, il est occulté par les appareils régulateurs qui dissimulent ou nient son existence; les sources d'information sur le sujet demeurent rares et difficilement accessibles, surtout si l'on veut éviter le risque d'un étiquetage comme lesbienne. Comparativement au Québec d'alors, dans des villes comme Paris, Amsterdam, Berlin, ou, plus près de Montréal, New York et Provincetown, il était nettement plus facile de dénicher livres, films et disques, d'oser se les procurer, d'obtenir de l'information sur des associations homophiles, de découvrir les préférences lesbiennes de telle chanteuse ou de telle comédienne et ainsi de suite. Ces représentations de l'homosexualité (féminine et masculine) alimentent la construction d'une identité lesbienne en fournissant des modèles et des légitimations de soi. En outre, elles font connaitre l'existence de sous-cultures dans d'autres villes que Montréal et suggèrent des destinations que certaines visiteront par la suite. Partager des connaissances et des références symboliques renforce également le sentiment d'appartenance à un même groupe minoritaire.

Enfin, le quart des femmes rencontrées a séjourné à l'étranger (quatre en France, une en Hollande et une aux États-Unis) pen- dant des périodes allant de quelques mois à quelques années, afin de voyager, d'étudier ou de travailler. Pour trois d'entre elles au moins, cet exil temporaire a eu un effet important sur leur processus d'identification comme lesbienne. Dans deux cas, la distanciation psychologique par rapport aux parents, l'expérience d'un milieu social davantage tolérant, le soutien accordé par des pairs (amies lesbiennes ou réseau homosexuel mixte) les ont amenées à s'affirmer dans leur différence sexuelle. Au retour, toutes deux ont décidé de quitter leur famille et de vivre leur lesbianisme.

\begin{abstract}
I had read about Holland. I looked for a place called the COC... I went there and it was like coming in out of a storm. It was just beautiful. This was a club for homosexuals, male and female, it was recreation, it was dancing. There was a lot of women there and they were very friendly, very helpful. And I learned a lot... because these women knew who they were, what they wanted and they taught me. To these women, it was quite normal, I was a human being.
\end{abstract}

Quant à la troisième, après une arrestation à la sortie d'un bar - expérience très douloureuse qui s'ajoutait à d'autres épisodes de répression - , elle quitte Montréal pour aller travailler aux États-Unis dans le domaine de l'hôtellerie ; là-bas, en s'insérant dans des réseaux homosexuels mixtes (elle travaille pour des employeurs gais et voyage avec eux pendant la morte saison), elle peut continuer à vivre ouvertement son lesbianisme tout en se plaçant à l'abri des sanctions venant de l'entourage immédiat.

Sauf lors d'un séjour prolongé à l'étranger, les femmes interviewées voyageaient le plus souvent en couples et ne cherchaient pas à nouer de nouvelles relations. Leur expérience des lieux de rencontre pour lesbiennes varie considérablement. À Paris, les « bars de femmes » étaient annoncés dans les guides d'information touristique. Certains étaient bien connus, comme Le monocle ou Chez Moune. Celles qui s'y sont aventurées ont été déçues : elles s'y sentaient mal à l'aise, peu habituées à côtoyer des lesbiennes qui s'affichaient par leurs vêtements et des comportements apparemment stéréotypés reproduisant les rôles masculins et féminins ; de plus, elles méconnaissaient les codes et craignaient de se faire «plumer» dans ces 
boîtes pour touristes. Ces femmes, pour la plupart des professionnelles, n'étaient pas non plus familières avec l'univers des bars montréalais - un monde qu'elles craignaient - et se risquaient à pénétrer dans un tel lieu pour la première fois de leur vie.

Celles qui ont visité des bars américains au cours des années 1960, principalement à Provincetown dont la popularité commençait à croître, ont eu des réactions mitigées. D'un côté, elles étaient fascinées de découvrir l'existence de milieux lesbiens dans un pays voisin; de l'autre, elles ne s'identifiaient pas toujours au style des lesbiennes qu'elles observaient :

Je ne me faisais pas regarder comme ici. C'était comme si j'avais le droit de vivre, il y en avait d'autres comme moi. C'était ça qui était important, c'est ça que je recherchais. J'étais pas toute seule au monde, je n'étais pas considérée comme une dépravée. Et puis il y avait la nature, la mer...

J'allais au bar de femmes. J'avais les yeux ronds. Je regardais fonctionner les couples de femmes américaines dont j'avais entendu parler dans les « paperbacks». C'était d'autres modèles de femmes. Elles étaient sûrement affirmées dans leur lesbianisme mais elles étaient toutes négligées et on n'était pas habituées à cela. On portait des pantalons mais c'est tout. Je ne crois pas que j'en portais pour aller travailler.

\section{LES ANNÉES P'TOWN ET APRÈS...}

La popularité de Provincetown va s'amplifier énormément durant les années 1970. La proximité géographique, l'accessibilité financière, la beauté des lieux, la variété des activités offertes, la présence d'une culture lesbienne-féministe, la qualité et la diversité des établissements pour gais et lesbiennes sont autant de facteurs qui ont contribué à sa notoriété. Outre la fréquentation des plages et des établissements spécialisés, le fait de pouvoir circuler dans la rue en tenant la main de son amante sans s'exposer à des sanctions, l'expérience d'être reconnue en tant que lesbienne tout en étant traitée avec respect dans les commerces et les autres lieux publics, la possibilité d'échanger des re-

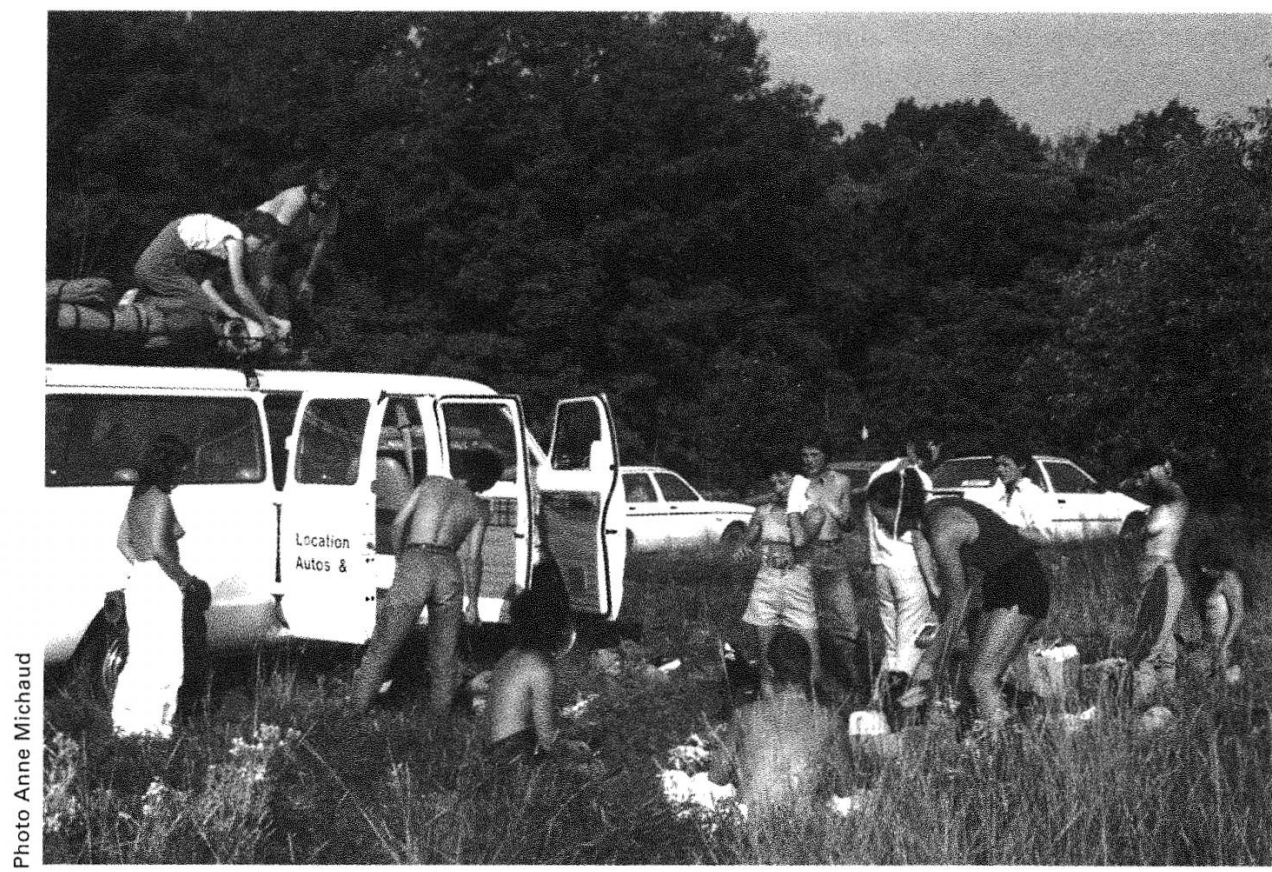

Un groupe de Québécoises arrivant au Festival de musique de femmes au Michigan dans les années 1980.

gards de reconnaissance, voire de séduction, de l'une à l' autre ${ }^{8}$, constituent autant d'expériences plaisantes et stimulantes qui renforcent positivement l'identité lesbienne. Oasis de liberté, Provincetown fournit la possibilité unique d'exister socialement en tant que lesbienne, de prendre place dans la cité.

Depuis cette décennie, les activités touristiques pratiquées par les lesbiennes se sont multipliées et diversifiées, s'adaptant à la pluralité des goûts, des moyens financiers, voire des tendances politiques. Avec la plus grande visibilité des sous-cultures homosexuelles dans les grandes villes, visiter un bar, bouquiner dans une librairie spécialisée, flâner dans un quartier lesbien ou gai, photographier une place, un monument commémorant un personnage ou un événement marquant de l'histoire des lesbiennes et des gais sont devenus des rituels pratiqués, lors de leurs voyages, par des milliers de lesbiennes d'ici et d'ailleurs. Les nombreuses rencontres internationales, à caractère politique, scientifique, culturel ou sportif, fournissent également l'occasion d'explorer diverses destinations touristiques et stimulent les échanges avec des pairs d'autres pays. Mentionnons, à titre d'exemple, la sixième édition des Jeux gais qui se déroulera à Sydney en 2002, après San Francisco (deux fois), Vancouver, New York et Amsterdam. Les ressources touristiques s'adressant spécifiquement aux lesbiennes, organisées sur une base associative ou, le plus souvent, commerciale, se sont considérablement accrues. Outre la possibilité de voyager en compagnie exclusive d'autres lesbiennes, elles offrent des circuits alternatifs et des hébergements conviviaux dans les destinations courantes. La revue Lesbian Connection fournit un aperçu de la variété des possibilités : retraite artistique sur une terre possédée collectivement par des femmes dans les montagnes de l'état de New York, couette et café à Florence, croisières organisées en Grèce et au Moyen-Orient par la compagnie Olivia, échange d'appartement, camping sauvage en Alaska avec des guides lesbiennes, etc. Les Amazones des grands espaces organisent des activités de plein air dans la région montréalaise et des excursions en province depuis plusieurs années (« Dossier Sports et plein air », 1998).

Alors que les gais ont développé des circuits de « partys » dans les villes, c'est en dehors des grands centres et à l'abri des intrus que des lesbiennes, féministes et radicales, ont créé de toutes pièces des méga-événements où peut se déployer une culture lesbienne. Les festivals de musique de femmes en sont le meilleur exemple. Celui du Michigan, le plus fréquenté par les Québécoises, attire bon an mal an de 5000 à 10000 lesbiennes et fêtera cette année son vingt-cinquième anniversaire. 
La riche histoire de ce festival reflète celle des solidarités et des divisions qui ont marqué le mouvement des lesbiennes en Amérique du Nord (Morris, 1999).

Des recherches sont nécessaires afin de mieux cerner les pratiques touristiques contemporaines chez les lesbiennes. Pour ce qui est de la période étudiée dans cet article, soit les années 1950 et 1960 , l'analyse des parcours individuels fait nettement ressortir, malgré le faible nombre de témoignages, l'importance de la mobilité spatiale et géographique comme un trait caractéristique des trajectoires lesbiennes. Cette mobilité se traduit notamment par des phénomènes migratoires, dont la direction et la fréquence demandent à être examinées de plus près, ainsi que par la pratique d'activités touristiques nombreuses et variées, dont l'intensité n'a fait qu'augmenter au cours des dernières décennies. Les deux principales motivations sous-jacentes à cette mobilité sont la volonté d'échapper au contrôle de l'entourage et de gagner une marge d'autonomie personnelle afin de vivre son lesbianisme, de même que l'expression d'une identité propre qui se nourrira de la rencontre avec des femmes (et des hommes) semblables à soi, des modèles inventés dans d'autres contrées, des cultures homosexuelles actuelles et passées. On peut penser qu'aujourd'hui une telle affirmation identitaire revêt une importance prépondérante lors des nombreux périples qu'entreprennent les lesbiennes. Sur de multiples chemins, elles poursuivent leur quête d'un ailleurs, réel et imaginaire, qui leur donne le goût d'un meilleur « ici et maintenant $»$.

Détentrice d'un doctorat en sociologie et professeure au Collège de Maisonneuve, Line Chamberland a dirigé en 1997 le numéro thématique "Homosexualités : enjeux scientifiques et militants » de la revue Sociologie et sociétés.

Un comité de lecture a lu et accepté ce texte

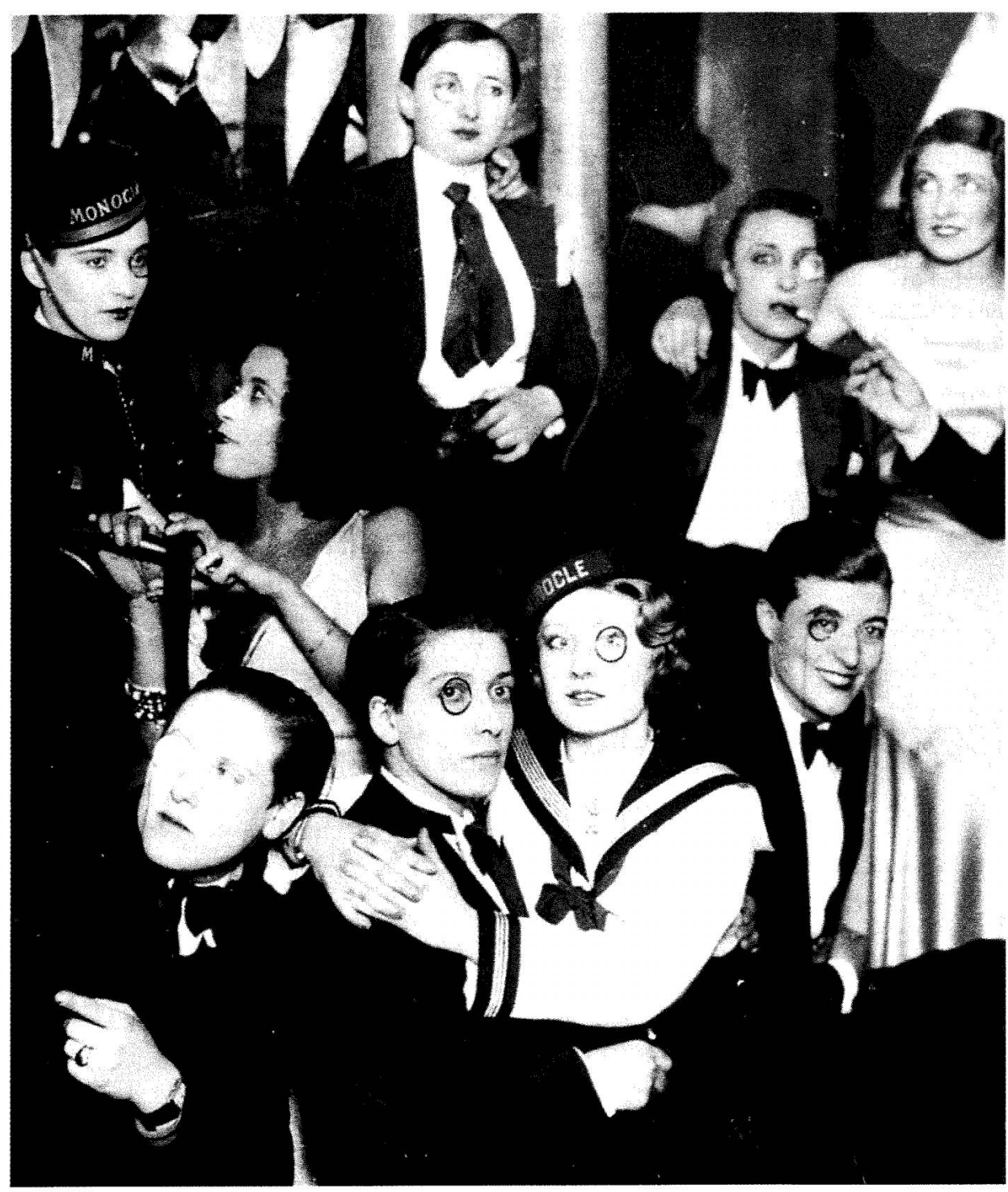

Paris était déjà dans les années 1920 une ville ouverte à la diversité sexuelle. Ici, des lesbiennes au bar Le Monocle. (Bibliothèque Historique de Paris, Fonds France-Soir, tiré de Les garçonnes, modes et fantasmes des Années folles de Christine Bard paru en 1998 chez Flammarion, p. 6)

\section{NOTES}

1 Plusieurs chercheurs ont noté le phénomène de migration des populations homosexuelles vers les grands centres ainsi que le groupement des espaces commerciaux et associatifs dans certains quartiers, auquel peut s'ajouter une relative concentration résidentielle, constituant ainsi des « villages gais » (Bell et Valentine, 1995 ; D'Emilio, 1983 ; Kennedy et Davies, 1995 ; Kinsman, 1996 ; Lewin, 1996). À Montréal, tout comme dans d'autres villes, les communautés gaies et lesbiennes se sont le plus souvent localisées dans des périmètres distincts. Les lieux pour lesbiennes sont également plus disséminés et moins visibles dans l'espace urbain. Pour une discussion des différences entre gais et lesbiennes dans l'occupation du territoire urbain, voir l'article de Tamar Rothenberg, «'And She Told Two Friends'. Lesbians
Creating Urban Social Space » (Bell et Valentine, 1995: 165-168).

2 Le pouvoir d'achat des lesbiennes, dont les revenus sont généralement inférieurs étant donné la moyenne des salaires féminins, n'a pas reçu la même attention médiatique. Il n'existe malheureusement aucune étude empirique québécoise sur la situation économique des lesbiennes. La constitution d'un marché gai dans une économie capitaliste accroît la visibilité et le pouvoir politique des homosexuels. Toutefois, ce phénomène soulève aussi un questionnement dans les associations militantes : en effet, il occulte les inégalités liées à la classe sociale et au sexe parmi cette population et risque de renforcer les divisions au sein du mouvement des gais et des lesbiennes (Strub, 1997 ; Gluckman et Reed, 1997). 
3 Pour un bref aperçu des recherches sur les lesbiennes effectuées au Québec de 1980 à 1997, en langue française, on pourra consulter Chamberland (1999).

4 Pour une présentation plus détaillée de cette problématique théorique, voir le premier chapitre de mon livre Mémoires lesbiennes. Le lesbianisme à Montréal entre 1950 et 1972 (1996) ainsi que les chapitre 4,5 et 6 qui portent respectivement sur l'analyse du contrôle judiciaire, religieux et psychomédical exercé sur les lesbiennes pendant cette période.

5 Au moment des entrevues, ces femmes étaient âgées de 39 à 65 ans. L'échantillon retenu se caractérisait à la fois par des traits communs à toutes les narratrices ou presque (langue française, éducation catholique) et par sa diversité quant à leur origine sociale et géographique, leur niveau de scolarité et les emplois qu'elles occupaient ou avaient occupés : enseignante, infirmière, journaliste, bibliothécaire, secrétaire, commis-vendeuse, employée de manufacture, serveuse, chanteuse, conductrice de camions, etc. Il a été constitué à l'aide d'une technique d'échantillonnage en boule-de-neige appliquée à partir de plusieurs chaînes de contact initiales afin de maximiser sa diversité. La méthode employée est l'analyse de récits autobiographiques selon divers axes thématiques et en lien avec le contexte socio-historique de l'époque. Voir Chamberland (1996).

6 Les interviewées qui ont été mariées pendant une durée variable ne disposaient effectivement d'aucune autonomie pour faire des voyages seules au cours de cette période de leur vie. Il en va de même pour la seule interviewée qui avait vécu dans une communauté religieuse. Celle-ci rapporte toutefois avoir profité de ses séjours dans des résidences d'été, là où la surveillance était moins étroite, pour se promener dans les boisés avec sa « grande amie »... Sur le rapport des lesbiennes à l'espace public dans le contexte montréalais des années 1950 et 1960 , voir mon article «La conquête d'un espace public : Les bars fréquentés par les lesbiennes » (Demczuk et Remiggi, 1998 : 129-164).

7 Fondé en 1946, le COC avait entre autres comme but d'organiser des activités sociales dans un cadre sécuritaire. Pour démontrer la moralité des personnes homosexuelles et obtenir pour elles un traitement égal à celui des autres citoyens, le COC condamnait le travestisme et s'opposait à tout laxisme sexuel. De son côté, notre interviewée - une professionnelle instruite - a particulièrement apprécié l'accueil qu'on lui a fait et la convivialité au sein de l'association. Cette narratrice, la seule d'origine anglophone, a voyagé en Hollande et en Allemagne alors que les narratrices francophones, surtout parmi les plus instruites, privilégiaient la France comme principale destination.
8 Dans son article intitulé « The Lesbian Flâneur », Sally Munt évoque son expérience personnelle de la ville de Brighton, capitale gaie du sud de l'Angleterre: «Brighton introduced me to the dyke stare, it gave me permission to stare. It made me feel I was worth staring at, and I learned to dress for the occasion. Brighton constructed my lesbian identity, one that was given to me by the glance of others, exchanged by the looks I gave them, passing - or not passing - in the street» (Bell et Valentine, 1995: 115).

\section{BIBLIOGRAPHIE}

Bell, David, et Gill Valentine (1995), Mapping Desire. Geographies of Sexualities, London, Routledge.

Chamberland, Line (1999), «La lente émergence du champ des études lesbiennes en langue française », sous la direction de Denise Veilleux, La recherche sur les lesbiennes. Enjeux théoriques, méthodologiques et politiques. Voix féministes/Feminist Voices, $n^{\circ} 7$, Ottawa, Canadian Research Institute for the Advancement of Women/Institut canadien de recherches sur les femmes.

Chamberland, Line (1996), Mémoires lesbiennes. Le lesbianisme à Montréal entre 1950 et 1972, Montréal, Les Éditions du remue-ménage.

D'Emilio, John (1983), Sexual Politics, Sexual Communities. The Making of a Homosexual Minority in the United Stated, 1940-1970, Chicago, The University of Chicago Press.

Demczuk, Irène, et Frank W. Remiggi (sous la direction de) (1998), Sortir de l'ombre. Histoires des communautés lesbienne et gaie de Montréal, Montréal, VLB éditeur.

«Dossier Sports et plein air » (1998), revue Treize, 15, (2), automne, p. 15-24.

Faderman, Lillian (1991), Odd Girls and Twilight Lovers. A History of Lesbian Life in the Twentieth-Century America, New York, Penguin Books.

Gluckman, Amy, et Betsy Reed (1997), « The Gay Marketing Moment », sous la direction de Martin Duberman, A Queer World. The Center for Lesbian and Gay Studies Reader, New York, New York University Press, p. 519-525.

Higgins, Ross (1999), De la clandestinité à l'affirmation. Pour une histoire de la communauté gaie montréalaise, Montréal, Comeau et Nadeau.

Higgins, Ross (1997), Sense of Belonging: PreLiberation Space, Symbolics and Leadership in Gay Montreal, thèse de doctorat, Département d'anthropologie, Université McGill.

Hildebran, Andrea L. (1997), Lesbian Activism in Montreal : 1973-1979, mémoire de maîtrise en histoire, Université du Québec à Montréal.

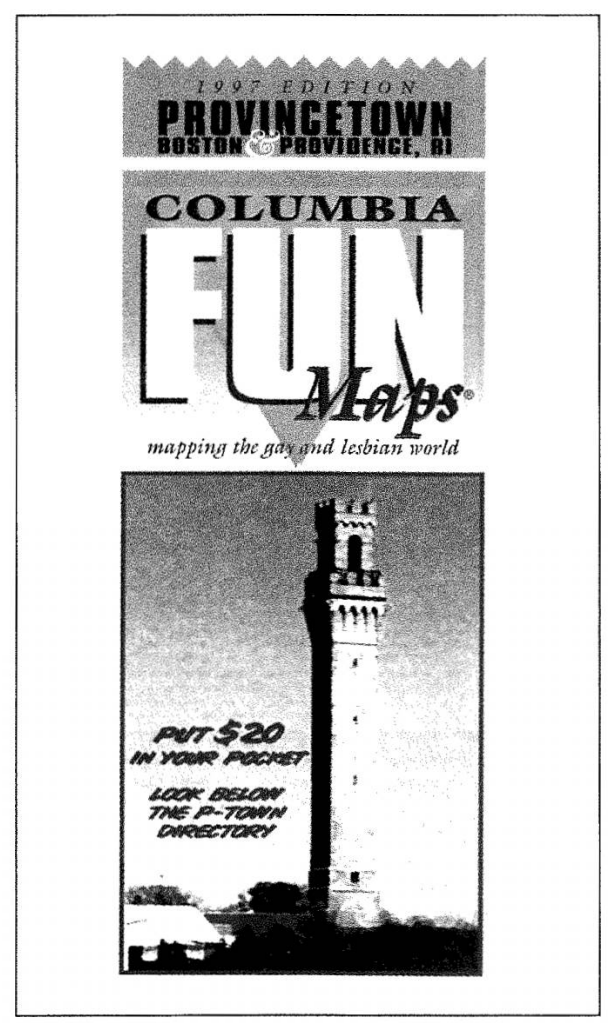

La tour commémore le débarquement en 1620 de pèlerins fuyant les répressions religieuses en Angleterre.

Kennedy, Elisabeth L., et Madeline D. Davies (1995), Boots of Leather, Slippers of Gold. The History of a Lesbian Community, New York, Routledge.

Kinsman, Gary (1996), The Regulation of Desire: Homo and Hetero Sexualities, $2^{\mathrm{e}}$ éd. révisée, Montréal, Black Rose Books.

Lewin, Ellen (sous la direction de) (1996), Inventing Lesbian Cultures in America, Boston, Beacon Press.

Morris, Bonnie J. (1999), Eden Built by Eves. The Culture of Women's Music Festivals, Los Angeles, Alyson Books.

Newton, Esther (1993), Cherry Grove, Fire Island. Sixty Years in America's First Gay and Lesbian Town, Boston, Beacon Press.

Strub, Sean (1997), « The Growth of the Gay and Lesbian Market », sous la direction de Martin Duberman, A Queer World. The Center for Lesbian and Gay Studies Reader, New York, New York University Press, p. 514-518.

\section{REMERCIEMENTS}

Je remercie Monik Audet, Nicole Lacelle et Ross Higgins pour leurs commentaires sur une version préliminaire de ce texte. 